\title{
Light Curves of Be Stars due to Orbiting Arcs of Matter or Prominence-Like Structures
}

\section{J. Zorec}

Institut d'Astrophysique de Paris, CNRS, 98 bis Bd. Arago, F-75014

Paris, France

\section{Y. Frémat}

\section{Université de Mons-Hainaut, Belgique}

\author{
A.M. Hubert \\ Observatoire de Paris-Meudon, DASGAL/UMR 8633 du CNRS, \\ F-92195 Meudon Principal Cedex, France
}

\begin{abstract}
An alternative explanation to the light variations of some Be stars observed during their $\mathrm{Be} \rightleftharpoons \mathrm{Be}$-shell phase changes is proposed in terms of orbiting or passing clouds. Discrete ejections of matter and/or the presence of orbiting clouds suggest that the circumstellar envelopes of these Be stars may be clumpy.
\end{abstract}

\section{Introduction}

Studies of line spectra, mostly those concerning the He I 6678 line accumulate evidence for discrete ejections taking place in Be stars (Smith et al. 1991, 1993, Floquet et al. 1999). On the other hand, short and long-lived outbursts observed during the HIPPARCOS photometric survey can also be interpreted as effects due to ejected layers or slabs, which gradually become diluted. Finally, longterm spectrophotometric variations of Be stars seem also to correspond to several scenarios implying ejections of massive layers (Moujtahid et al. 1999). Thus, it cannot be ruled out that phenomena similar to some huge solar-like filaments (prominences) exist in Be stars. In this paper we present numerical simulations of light variations produced by long-lived orbiting prominence-like matter.

\section{The model}

Let us assume that a filament, a piece of a ring, arc of matter or cloud, of angular extension $\Delta \Phi$ as measured from the star, is ejected. At the moment, we also assume that this arc of matter, or cloud, does not dissipate before it completes an orbit around the star. For simplicity, it is assumed that the arc moves in a circular orbit on a plane that contains the line of view. It can then be realized that an arc, which along its movement preserves its radial opacity $\tau$, its angular extent $\Delta \Phi$ and its temperature, produces a symmetrical light curve 

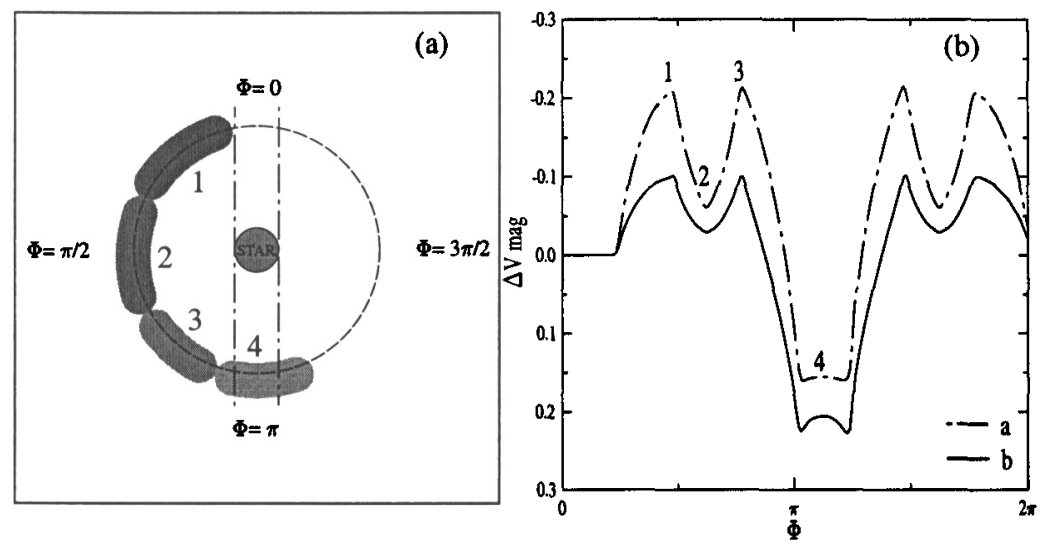

Figure 1. (a) Arc of matter moving around the star in a circular orbit from position 1 to 4 towards phase $\Phi=2 \pi$ (we assumed variable physical conditions in the arc of matter). (b) Light curves given in difference of magnitudes $\Delta V=V-V_{*}$ produced when the arc of matter moves from 1 to 4 and its symmetrical extrapolation for the remaining half orbit. The physical characteristics of the cloud in Fig. 1b are assumed not to change during the movement and they are given in Table 1. (1) and (3): emission phases, (2): self-absorption phase, (4): deep absorption phase.

with respect to the phase the arc passes through the sight meridian. On the other hand, clouds with variable physical characteristics produce asymmetrical light curves.

Table 1. Physical parameters of ejected arcs of matter

\begin{tabular}{cccccc} 
Curve & $\mathrm{S}$ & $\Delta R$ & tau & $\Delta \Phi$ & $H_{z}$ \\
\hline $\mathrm{a}$ & 0.75 & $0.5 R_{*}$ & 0.5 & $\pi / 4$ & $0.75 R_{*}$ \\
$\mathrm{~b}$ & 0.75 & $0.5 R_{*}$ & 1.5 & $\pi / 4$ & $0.75 R_{*}$
\end{tabular}

\section{Results}

In Fig. 1a is sketched the orbital movement of an arc of ejected matter of variable physical conditions, as expected for most real cases. The phase of the movement is $\Phi=0$ at the arc merging, which was assumed to occur behind the star. For the sake of a simple explanation of the light variation mechanism, in Fig. 1b are shown, however, the light curves as a function of the phase $\Phi$, produced by moving arcs of matter of constant physical characteristics which are given in Table 1. In Table 1 we have: $S$ the source function of continuum radiation in the arc normalized to the stellar flux; the angular extent $\Delta \Phi$ of the arc; the distance $\Delta R$ from the stellar surface; the total opacity in the continuum at $\lambda=0.56 \mu \mathrm{m}$ 

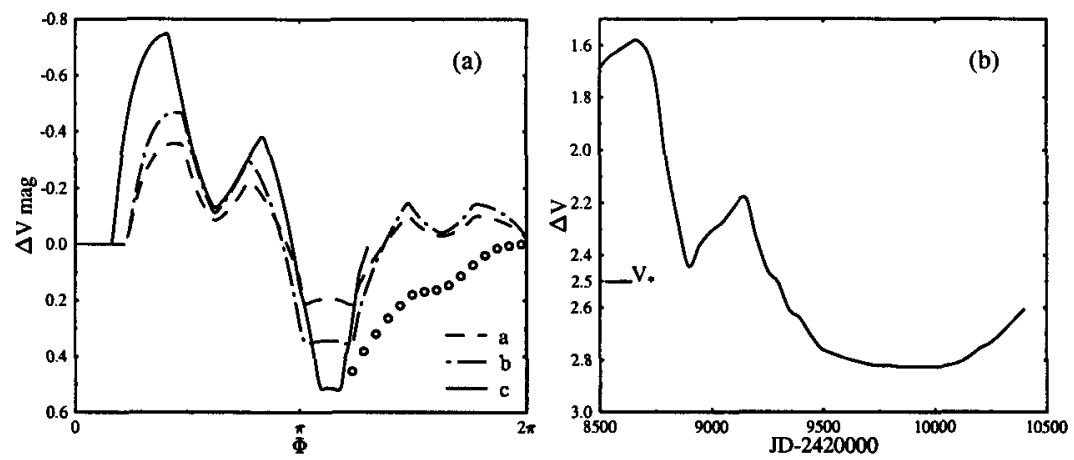

Figure 2. (a) Light curves produced by clouds of matter moving in orbits as in Fig. 1(a) having variable mean temperatures and densities (see Table 2). o : light curve produced by a fading cloud which disappears at phase $\Phi=2 \pi$. (b) Observed light curve in $\gamma$ Cas; $V_{*}$ is the apparent visual magnitude of the central star derived from its BCD spectrophotometric parameters and the HIPPARCOS parallax.

and the height $H_{z}$ of the cloud. For this particular case, the light curves also have two maxima at each half orbital period: the first maximum (point 1 in Fig. 1b) is due to the merging of the prominence; the second maximum (point 3 in Fig. 1b) appears when the cloud shows full exposure towards the observer, just before it crosses the stellar disc. Between the two light maxima there is a secondary light minimum (point 2 in Fig. 1b) as the front half of the arc absorbs the energy emitted by its rear half. The passing of the ring by the star produces either a light maximum or a light minimum (point 4 in Fig. 1b), depending on the values of the source function in the cloud, its opacity and the area of stellar disc covered by the ring.

Table 2. Variable parameters of ejected arcs of matter

\begin{tabular}{cccccccc} 
Curve & $\mathrm{S}_{i}$ & $\mathrm{~S}_{f}$ & $\Delta R$ & $\tau_{i}$ & $\tau_{f}$ & $\Delta \Phi$ & $H_{z}$ \\
\hline a & 2.0 & 0.5 & $0.5 R_{*}$ & 1.5 & 0.5 & $\pi / 4$ & $0.75 R_{*}$ \\
b & 2.5 & 0.5 & $0.5 R_{*}$ & 2.5 & 1.0 & $\pi / 4$ & $0.75 R_{*}$ \\
c & 2.5 & 0.5 & $1.0 R_{*}$ & 2.5 & 1.0 & $\pi / 4$ & $0.75 R_{*}$
\end{tabular}

We may expect that the ejection of an arc of matter is the result of some energetic phenomenon taking place in the stellar surface. We may then assume that the initial temperature of the ejecta is high. The arc may then cool down as it moves along its "orbit" and disperses somewhat. This implies that the value of the source function $S$ and that of the radial opacity are consequently reduced. For the sake of a simple numerical simulation, we assume that temperature and opacity vary linearly with the phase of the movement. It is assumed that the final values of the source function and opacity are attained at $\Phi=\pi$, and that they remain constant from $\Phi=\pi$ to $\Phi=2 \pi$. Table 2 gives the parameters of arcs which produce the light curves shown in Fig. 2a. The subindex " $i$ " stands 
for the initial values and " $f$ " for the final ones. The initial values of S correspond to temperatures approaching $50000 \mathrm{~K}$, suited for stars like $\gamma$ Cas and 59 Cyg. The pointed curve in Fig. 2a shows the light curve due to a fading cloud which disappears at $\Phi=2 \pi$.

\section{Discussion}

The opacities and distance scales used in these calculations are not far from those in current models of circumstellar layers that produce the visible continuum spectrum in Be stars. We see however that the ejected arcs of matter may easily produce light curves whose variation amplitudes resemble those observed during the exceptional Be-B-Be shell phase changes of stars like $\gamma$ Cas and 59 Cyg. For comparison with results presented in Fig $2 \mathrm{a}$, we show in Fig. $2 \mathrm{~b}$ the light curve of $\gamma$ Cas during its huge 1937-1942 variation. The apparent $V_{*}$ magnitude of the central star of $\gamma$ Cas was derived using the BCD (Paris spectrophotometric system) parameters and its HIPPARCOS parallax.

A further natural generalisation of the present model should imply a parabolic or an elliptical orbit of the ejected matter. Nevertheless, despite the approximate nature of our simulations, the shape of light curves obtained closely resemble those observed. The long period of light increase from the deep absorption observed in $\gamma$ Cas after its minimum in 1940-41 can be interpreted as due to a simultaneous change in the size and optical depth of the cloud as it disperses. A previous interpretation of such light curves was done by Hummel (1998) in terms of a temporarily tilted circumstellar disc. However, the predicted light maxima seem to be inverted as compared to observations. The variation of spectral line profiles as a function of the position of the arc around the central star is in progress. A consequence of the interpretation given in this paper to the light curve of Be stars during their phase changes is that the ejected parcels then need to pile up building up the circumstellar environment usually called disc or circumstellar envelope. It must then be expected that the density structure of these envelopes are either quite irregular or clumpy.

\section{References}

Floquet, M., Hubert, A.M., Hirata, R. et al. 1999, A\&A (to be submitted) Hubert, A.M., Floquet, M. 1998, A\&A 335, 565

Hummel, W. 1998, A\&A 330, 243

Moujtahid, A., Zorec, J., Hubert, A.M. 1999, A\&A 349, 151

Smith, M., Peters, G., Grady, C.A. 1991, ApJ 367, 302

Smith, M., Polidan, R. 1993, ApJ 408, 323 